\title{
Multiple Deletions in Mitochondrial DNA in a Patient with Progressive External Ophthalmoplegia, Leukoencephalopathy and Hypogonadism
}

\author{
Yuko Ohnuki ${ }^{1,2}$, Kazumi Takahashi ${ }^{2,3}$, Eri Iijima ${ }^{4}$, Wakoh Takahashi ${ }^{4}$, Shingo Suzuki $^{1}$, \\ Yuki Ozaki ${ }^{1}$, Ruriko Kitao ${ }^{5}$, Masatoshi Mihara ${ }^{5}$, Tadayuki Ishihara ${ }^{5}$, Michiyo Nakamura ${ }^{6}$, \\ Yoshie Sawano $^{6}$, Yu-ichi Goto ${ }^{6}$, Shunichiro Izumi ${ }^{2,3}$, Jerzy K. Kulski ${ }^{1,7}$, \\ Takashi Shiina $^{1}$ and Shunya Takizawa ${ }^{4}$
}

\begin{abstract}
Progressive external ophthalmoplegia (PEO) is one of a number of major types of mitochondrial disorders. Most sporadic PEO patients have a heteroplasmic large deletion of mitochondrial DNA (mtDNA) in the mitochondria in skeletal muscles. We herein analyzed mtDNA deletions using sub-cloning and Sanger sequencing of PCR products in a 31-year-old Japanese man with multiple symptoms, including PEO, muscle weakness, hearing loss, leukoencephalopathy and hypogonadism. A large number of multiple deletions was detected, as well as four kinds of deletion breakpoints identified in different locations, including m.3347 12322, m.5818_13964, m.5829_13964 and m.5837_13503.
\end{abstract}

Key words: mitochondrial DNA (mtDNA), multiple deletion, progressive external ophthalmoplegia (PEO), hypogonadism, leukoencephalopathy

(Intern Med 53: 1365-1369, 2014)

(DOI: 10.2169/internalmedicine.53.1320)

\section{Introduction}

Mitochondrial DNA (mtDNA) deletion syndromes comprise three major phenotypes: progressive external ophthalmoplegia (PEO), Kearns-Sayre syndrome (KSS) and Pearson syndrome. PEO is conventionally defined as progressive limitation of eye movements with normal pupils and ptosis of the eyelids. KSS is a multisystem disorder with the following symptoms: cardiac conduction block, pigmentary retinopathy and PEO. Pearson syndrome is characterized by sideroblastic anemia and exocrine pancreas dysfunction and is usually fatal in infancy. A few individuals with PEO have other manifestations of KSS but do not fulfill all of the clinical criteria for the diagnosis. This condition is called "PEO plus" in GeneReviews at the GeneTests Medical Genetics Information Resource (1). Most sporadic PEO patients have a heteroplasmic large deletion of mtDNA in the muscle. In patients with multiple mtDNA deletions, additional clinical features may be present, such as sensory axonal neuropathy, optic atrophy, ataxia, hypogonadism and parkinsonism $(2,3)$. Mutations in nuclear genes, such as POLG1 and POLG2 (DNA polymerase gamma) (4, 5), PEO1 (the helicase Twinkle) (6) and SLC25A4 (other alias: ANT1, the adenine nucleotide translocator) (7), have thus far been identified as driving multiple mtDNA deletion genes in PEO patients $(2,3)$. These proteins are involved in mtDNA replication and transcription, and their functional loss results in the

${ }^{1}$ Department of Molecular Life Science, Basic Medical Science and Molecular Medicine, Tokai University School of Medicine, Japan, ${ }^{2}$ Department of Clinical Genetics, Tokai University Hospital, Japan, ${ }^{3}$ Department of Obstetrics and Gynecology, Tokai University School of Medicine, Japan, ${ }^{4}$ Department of Internal Medicine, Division of Neurology, Tokai University School of Medicine, Japan, ${ }^{5}$ Department of Neurology, National Hakone Hospital, Japan, ${ }^{6}$ Department of Mental Retardation and Birth Defect Research, National Center of Neurology and Psychiatry, Japan and ${ }^{7}$ Centre for Forensic Science, The University of Western Australia, Australia

Received for publication July 8, 2013; Accepted for publication October 27, 2013

Correspondence to Dr. Yuko Ohnuki, yukom@is.icc.u-tokai.ac.jp 
secondary accumulation of abnormal-sized mtDNA (3). In this report, we identified breakpoints of mtDNA deletions using sub-cloning and Sanger sequencing of polymerase chain reaction (PCR) products in a 31-year-old Japanese man with multiple symptoms, including PEO, muscle weakness, hearing loss, leukoencephalopathy and hypogonadism.

\section{Case Report}

The patient was initially diagnosed with IgA nephropathy, hypogonadism and hearing loss at 24 years of age. He noticed double vision and a gait disturbance; these symptoms gradually worsened at 30 years of age, and he was admitted to our hospital at 31 years of age. The patient's parents were not consanguineous. He had a younger sister who exhibited no symptoms. He had no family history of similar symptoms. He was $170 \mathrm{~cm}$ in height and $49 \mathrm{~kg}$ in weight. He had gynecomastia, bilateral ptosis and ophthalmoplegia with restriction of adduction. He also had mild muscle weakness in the limbs. Tendon reflexes were normal.

Laboratory examinations showed hypergonadotropic hypogonadism (testosterone: $0.13 \mathrm{ng} / \mathrm{mL}, \mathrm{LH}: 14.8 \mathrm{mIU} / \mathrm{mL}$, FSH: $6.14 \mathrm{mIU} / \mathrm{mL})$. Although the serum lactate $(12.6 \mathrm{mg} /$ $\mathrm{dL})$ and pyruvate $(0.6 \mathrm{mg} / \mathrm{dL})$ levels were normal, the lactate level was slightly elevated in the cerebral spinal fluid $(18.2 \mathrm{mg} / \mathrm{dL}$, compared to a normal level of $<18.0 \mathrm{mg} / \mathrm{dL})$. No aerobic exercise tests were performed. A chromosomal analysis showed that the patient's karyotype was 46,XY and that he did not have Klinefelter syndrome. An X-ray film of the chest showed a hilar shadow; however, there was not enough evidence of sarcoidosis. Fluid attenuated inversion recovery (FLAIR) brain magnetic resonance imaging (MRI) sequences (Fig. 1) showed diffuse leukoencephalopathy affecting both cerebral hemispheres, although the brain stem and cerebellum were spared. Needle electromyography (EMG) of the right first dorsal interosseous and right tibialis anterior muscles was normal. The patient's intelligence quotient (IQ) according to the Wechsler Adult Intelligence Scale III (WAIS-III) was estimated as follows: verbal $\mathrm{IQ}=85$, performance $\mathrm{IQ}=78$ and full-scale $\mathrm{IQ}=80$. Muscle biopsy specimens obtained from the left biceps showed brachii with many ragged-red fibers on a modified Gomori-trichrome stain (Fig. 2a, b). Succinate dehydrogenase (SDH) and cytochrome c oxidase (COX) staining were also examined and a COX deficiency was observed (Fig. 2c, d). Based on these findings, the patient was diagnosed with 'PEO plus;' therefore, we decided to analyze his mtDNA in detail in order to investigate and confirm the presence of multiple mtDNA deletions within the mitochondria.

\section{DNA analysis and results}

This study was approved by the ethics committee of Tokai University School of Medicine (11I-17). Informed consent was obtained from the patient and his mother to perform the genetic studies.

Genomic DNA was extracted directly from muscle tissue
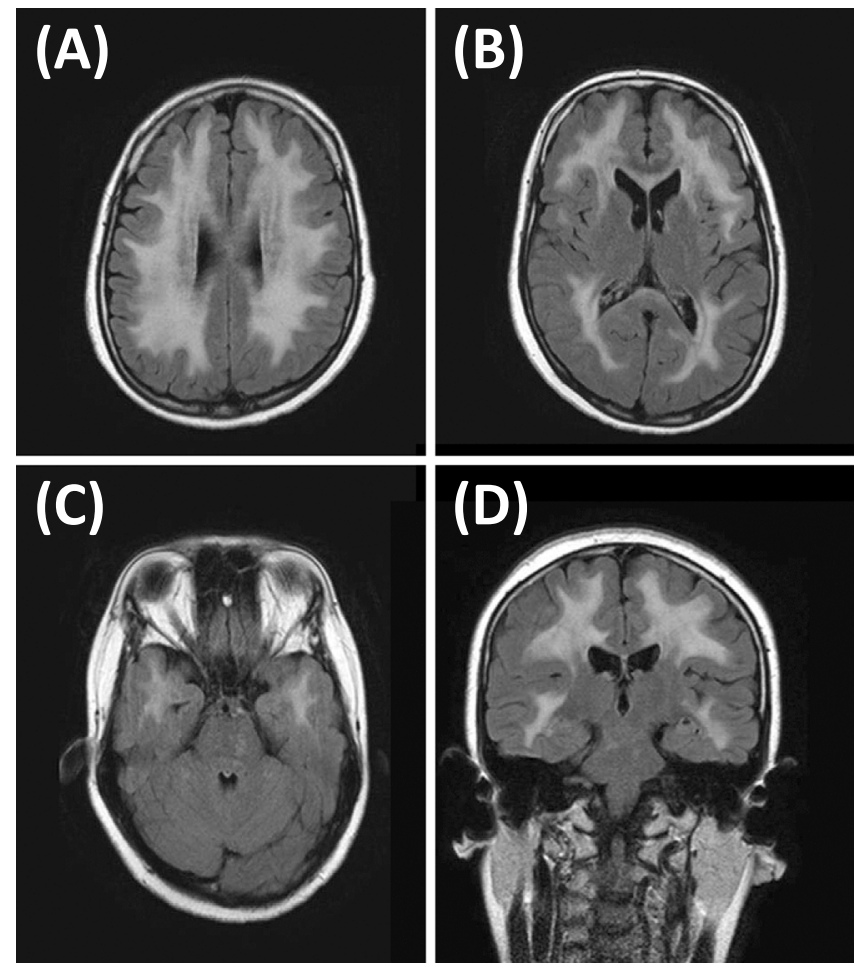

Figure 1. Brain MRI (FLAIR). (A-C) and (D) indicate MRI of axial and coronal sections, respectively. Symmetrical hyperintensity regions were observed in the bilateral cerebral white matter.

using the QIAamp DNA Investigator Kit (QIAGEN, Hilden, Germany). Whole mtDNA fragments, excluding the displacement loop (D-loop), were amplified via long-range PCR. A new set of mtDNA primers was designed for the conserved nucleotide sites provided by the GenBank database (www.ncbi.nlm.nih.gov) and to amplify sequences between the 12S rRNA gene and the Cytochrome $b$ gene (amplified size: 14,457 bp on mtDNA sequence (GenBank/ EMBL/DDBJ accession number: NC_012920) with the sense primer (mtDNA-F1: 5'-CAGCAATGCAGCTCAAAA CGCTTA-3') and the anti-sense primer (mtDNA-R2: 5'-GG CCTCGCCCGATGTGTAGGA-3'). The $20-\mu \mathrm{L}$ amplification reaction volume contained $10 \mathrm{ng}$ of genomic DNA, one unit of high-fidelity PrimeSTAR ${ }^{\circledR}$ GXL DNA Polymerase (TaKaRa BIO Inc., Otsu, Japan), 5xPCR buffer, $2.5 \mathrm{mM}$ of each dNTP and $0.5 \mu \mathrm{M}$ of each primer. The cycling parameters were as follows: 35 cycles of $98^{\circ} \mathrm{C} / 10 \mathrm{sec}, 60^{\circ} \mathrm{C} / 20 \mathrm{sec}$ and $68^{\circ} \mathrm{C} / 15 \mathrm{~min}$.

Based on our PCR analysis, we clearly detected evidence of PCR products from muscle DNA with multiple deletions within the mtDNAs in the electrophorese products. Namely, we observed the 15-kb wild-type mtDNA, 7-kb and 7.5-kb major deleted fragments and some minor deleted fragments ranging from $6 \mathrm{~kb}$ to $9 \mathrm{~kb}$ in the patient's genomic DNA (Fig. 3). These deletions were not observed in the patient's peripheral blood cells or the DNA obtained from the skeletal muscle and peripheral blood cells of control subjects.

In order to identify the breakpoints, we performed sub- 


\section{a}
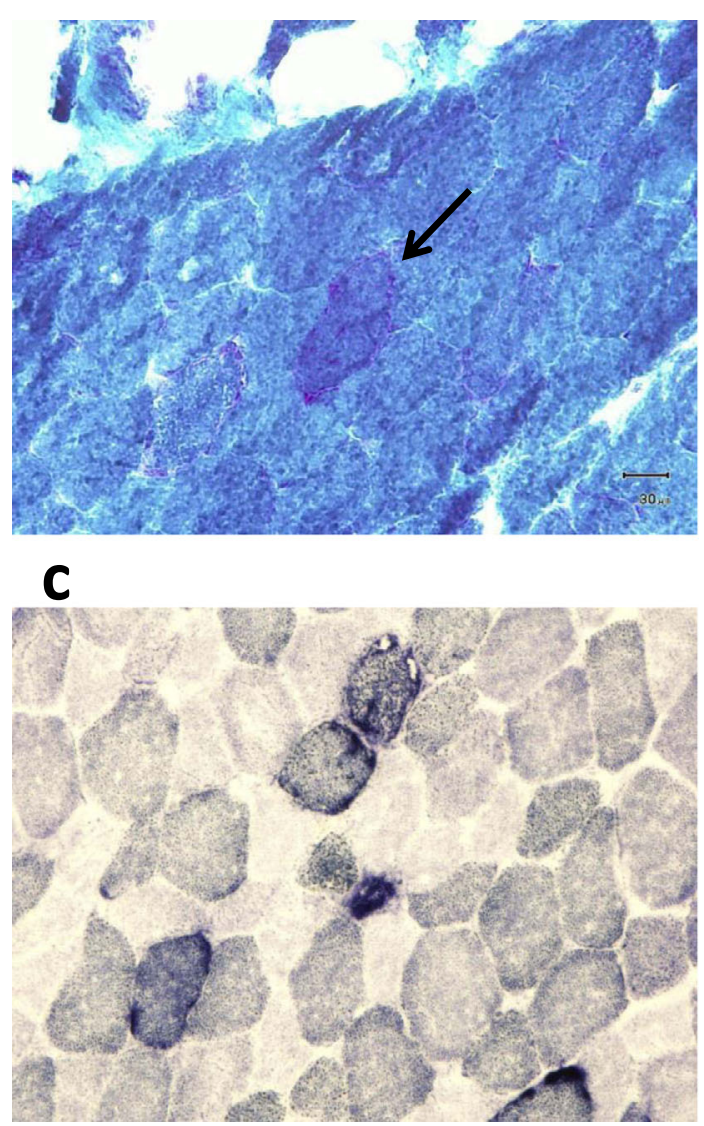

b

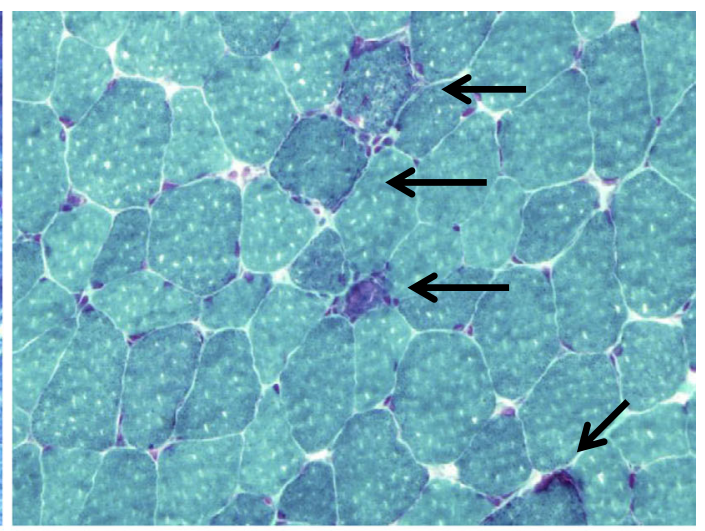

d

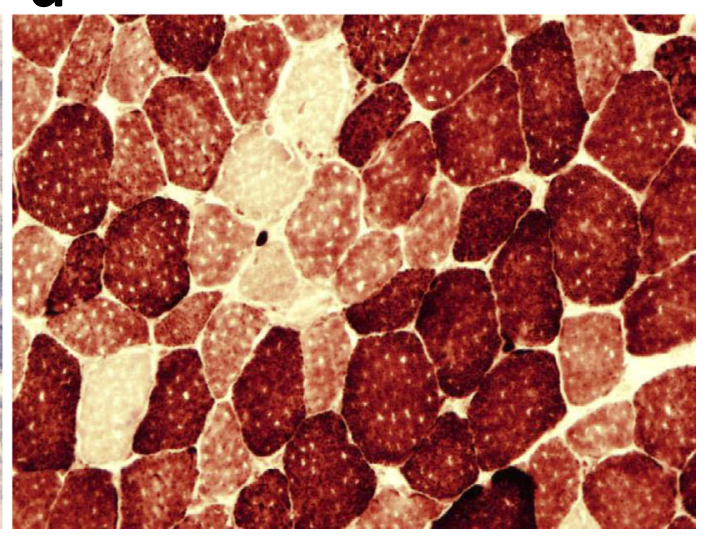

Figure 2. Biopsy specimen of the brachial biceps muscle stained with modified Gomori-trichrome staining (a, b). The arrow indicates so-called "ragged-red fibers." (c) SDH (Succinate Dehydrogenase) staining (d) COX (Cytochrome Oxidase) staining; a COX deficiency is observed.

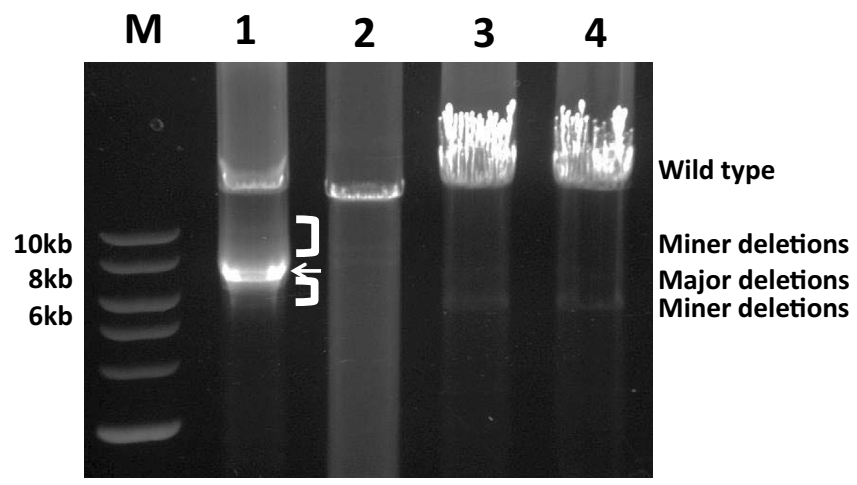

Figure 3. A long-range PCR analysis of the patient's mtDNA. The 15-kb fragments from nucleotide positions 767 to 15050 were amplified via long-range PCR using total tissue DNA extracted from the skeletal muscle (Lane 1) and peripheral blood cells (Lane 3) of the patient. Lanes 2 and 4 show PCR fragments derived from control skeletal muscle and control peripheral blood cells, respectively. "M" indicates $1 \mathrm{~kb}$ in the DNA ladder.

cloning and Sanger sequencing of the deleted DNA fragments. The targeted DNA fragments were isolated using a QIAquick gel extraction kit (QIAGEN). Sub-cloning and transformation of the fragments were performed according to the manufacturer's protocol for the TArget clone Kit (TOYOBO, Osaka, Japan) and DH5 $\alpha$-competent cells (TOYOBO). After sub-cloning, direct PCR was performed using the following parameters: 35 cycles of $98^{\circ} \mathrm{C} / 10 \mathrm{sec}$, $65^{\circ} \mathrm{C} / 20 \mathrm{sec}$ and $68^{\circ} \mathrm{C} / 7 \mathrm{~min}$. The nucleotide sequences randomly selected nine subclones that were directly sequenced using an ABI3130 genetic analyzer (Life Technologies, Carlsbad, USA) in accordance with the protocol of the Big Dye terminator method. We used various primers to detect the sequence breakpoints of the deletions according to the primer walking method (Table). The sequence-generated chromatogram data were analyzed using the Sequencher ver. 4.10 DNA sequence assembly software program (Gene Code Co., Ann Arbor, USA).

Using this procedure, four kinds of deletion breakpoints were identified, including m.3347_12322 (1), m.5818_ 13964 (2), m.5829_13964 (3) and m.5837_13503 (4), which corresponded to 8,976-bp, 8,147-bp, 8,136-bp and 7,667-bp deletions, respectively (Fig. 4). Of the nine PCR sub-clones, six had m.3347_12322 (1), while the others had m.5818_ 13964 (2), m.5829_13964 (3) and m.5837_13503 (4). Hence, m.3347_12322 (1) was the most predominant deletion type in this case. The deletion breakpoints did not occur 
Table. Primer List for Primer Walking Method of Sanger Direct-sequencing

\begin{tabular}{lll}
\hline Primer name & Location & Primer seqeunce (5' to 3') \\
\hline mt-F1 & mt.767_790 & CAGCAATGCAGCTCAAAACGCTTA \\
mt-F2 & mt.761_781 & AGCACGCAGCAATGCAGCTCA \\
mt-F3 & mt.858_881 & CTATACTAACCCCAGGGTTGGTCA \\
mt-F4 & mt.806_829 & CACGGGAAACAGCAGTGATTAACC \\
mt-F5 & mt.1345_1368 & GAGGTGGCAAGAAATGGGCTACAT \\
mt-F6 & mt.1764_1787 & CCTGGCGCAATAGATATAGTACCG \\
mt-F7 & mt.2250_2273 & CACTCCTCACACCCAATTGGACCA \\
mt-F8 & mt.2718_2741 & CGAGAAGACCCTATGGAGCTTTAA \\
mt-F9 & mt.3230_3253 & GTTAAGATGGCAGAGCCCGGTAAT \\
mt-F10 & mt.3649_3672 & TCAATCCTCTGATCAGGGTGAGCA \\
mt-F11 & mt.4179_4202 & AAACTTCCTACCACTCACCCTAGC \\
mt-F12 & mt.4526_4549 & CACAGCGCTAAGCTCGCACTGATT \\
mt-R1 & mt.15200_15223 & GTCTGTCCCAATGTATGGGATGGC \\
mt-R2 & mt.15030_15050 & GGCCTCGCCCGATGTGTAGGA \\
mt-R3 & mt.14978_15001 & ATTGGCGTGAAGGTAGCGGATGAT \\
mt-R4 & mt.14903_14926 & TGAGGCGTCTGGTGAGTAGTGCAT \\
mt-R5 & mt.14438_14461 & AGCGATGGCTATTGAGGAGTATCC \\
mt-R6 & mt.13985_14008 & GTCAGGTTAGGTCTAGGAGGAGTA \\
mt-R7 & mt.13538_13561 & GGGCTCAGGCGTTTGTGTATGATA \\
mt-R8 & mt.13069_13092 & TATAGTGCTTGAGTGGAGTAGGGC \\
mt-R9 & mt.12733_12756 & CAGTTGGAATAGGTTGTTAGCGGT \\
mt-R10 & mt.12345_12368 & GTTAGGGTGGTTATAGTAGTGTGC \\
mt-R11 & mt.12009_12032 & TGTTAATGTGGTGGGTGAGTGAGC \\
mt-R12 & mt.11633_11656 & TGTTACTACGAGGGCTATGTGGCT \\
\hline & &
\end{tabular}

within the repeated sequences.

\section{Discussion}

Progressive external syndrome (PEO) is a mitochondrial myopathy associated with ptosis, ophthalmoplegia and variably proximal limb weakness. PEO is almost never inherited, suggesting that this disorder is caused by de novo mtDNA deletions that occur in the mother's oocytes during germline development or in the embryo during embryogenesis (1). Chen et al. (8) showed that the "common deletion" (m.8470_13446del4977) accounts for $0.01-0.1 \%$ of the approximately 150,000 copies of mtDNAs in the human oocyte (8). Even if mtDNA deletions were present in human oocytes, a small mitochondrial bottleneck would eventually effectively filter out these deletions. On rare occasions, a "deleted" mtDNA may slip through the ancestral line. On the other hand, many familial cases have been reported with multiple deletions of mtDNA. Most patients suffer from PEO and may have nuclear genetic defects, with the accumulation of mtDNA deletions (9). These nuclear genes are known to play important roles in controlling mtDNA synthesis and maintenance. However, no mutations were detected in POLG and PEOI that are known to be nuclear genes affecting mtDNA deletions (data not shown).

Multiple mtDNA deletions are associated with many clinical phenotypes (9), including cardiomyopathy, recurrent myoglobinuria, ataxia plus ketoacidotic coma, multiple symmetric lipomatosis, myoclonus epilepsy with ragged red fibers and myopathy with multisystemic features except

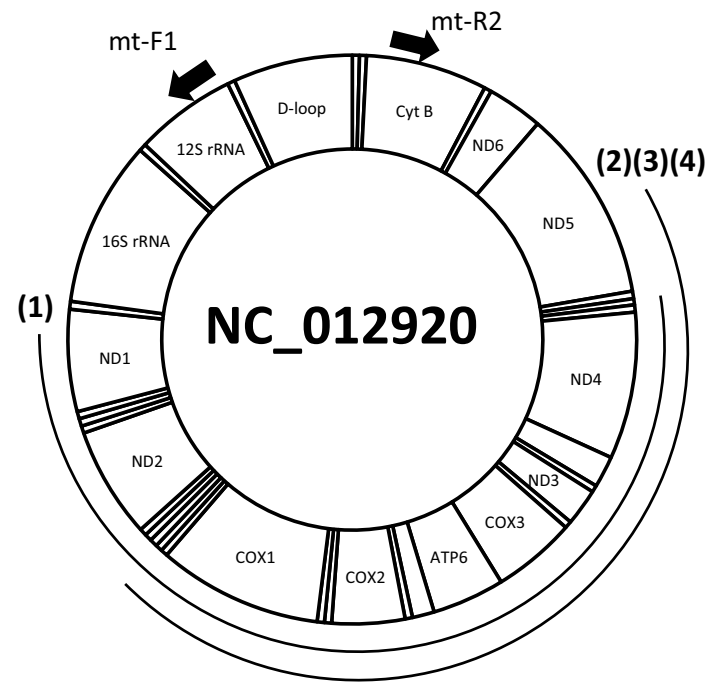

Figure 4. Locations of the mtDNA deletions. (1-4) indicate locations of four kinds of mtDNA deletions identified in this study such as m.3347_12322 (1), m.5818_13964 (2), m.5829_13964 (3), and m.5837_13503(4). Arrows indicate locations of long ranged PCR primers; mt-F1(mt.767_790) and mtR2 (mt.15030_15050).

PEO (9). We herein described a patient suffering from PEO, muscle weakness, hearing loss, leukoencephalopathy and hypogonadism. There are few previous case reports of patients with hypogonadism associated with PEO.

Luoma et al. (3) reported four families that were diagnosed with PEO in addition to premature menopause and parkinsonism. The authors recorded mutations in $P O L G$ in all of the families. In one of the families, the affected men exhibited testicular atrophy (3). These facts point to a defect in steroidogenesis, in which mitochondria play a role in regulating the serum steroid hormone concentrations $(3,10)$. Melberg et al. (11) also reported a family with autosomal dominant PEO and hypogonadism.

In the present case history, we followed the patient's symptoms for almost six years to eventually diagnose him with hypogonadism preceded by ptosis and ophthalmoplegia. Therefore, clinicians should take into consideration hypogonadism as a symptom of mitochondrial disorders, including PEO. Furthermore, multiple symptoms associated with PEO may be caused by nuclear genetic defects stemming from multiple mtDNA deletions that may be transmitted via Mendelian inheritance, which must be explained to patients and their families.

The authors state that they have no Conflict of Interest (COI).

\section{References}

1. DiMauro S, Hirano M. Mitochondrial DNA Deletion Syndromes. In: GeneReviews ${ }^{\circledR}$ at GeneTests: Medical Genetics Infromation Resource (database online). Copyright. Pagon RA, Adam MP, Ardinger HH, Bird TD, Dolan CR, Fong CT, Smith RJH, Stephens K, Eds. University of Washington, Seattle, May 2011: 
1993-2014. Available at http://www.genetests.org. Accessed June 11,2013

2. Ronchi D, Garone C, Bordoni A, et al. Next-generation sequencing revieals DGUOK mutations in adult patients with mitochondrial DNA multiple deletions. Brain 135: 3404-3415, 2012.

3. Luoma P, Melberg A, Rinne JO, et al. Parkinsonism, premature menopause, and mitochondrial DNA polymerase $\gamma$ mutations: clinical and molecular genetic study. Lancet 364: 875-882, 2004.

4. Van Goethem G, Dermaut B, Löfgren A, Martin JJ, Van Broeckhoven C. Mutation of POLG is associated with progressive external ophthalmoplegia characterized by mtDNA deletions. Nat Genet 28: 211-212, 2001.

5. Longley MJ, Clark S, Yu Wai, et al. Mutant POLG2 disrupts DNA polymerase gamma subunits and causes progressive external ophthalmoplegia. Am J Hum Genet 78: 1026-1034, 2006.

6. Spelbrink JN, Li FY, Tiranti V, et al. Human mitochondrial DNA deletions associated with mutations in the gene encoding Twinkle, a phage $\mathrm{T} 7$ gene 4-like protein localized in mitochondria. Nat
Genet 28: 223-231, 2001.

7. Kaukonen J, Juselius JK, Tiranti V, et al. Role of adenine nucleotide translocator 1 in mtDNA maintenance. Science 289: 782-785, 2000.

8. Chen X, Prosser R, Simonetti S, Sadlock J, Jagiello G, Schon EA. Rearranged mitochondrial genomes are present in human oocytes. Am J Hum Genet 57: 239-247, 1995.

9. Van Goethem G, Martin JJ, Van Broeckhoven C. Progressive external ophthalmoplegia characterized by multiple deletions of mitochondrial DNA: unraveling the pathogenesis of human mitochondrial DNA instability and the initiation of a genetic classification. Neuromolecular Med 3: 129-146, 2003.

10. Bose H, Lingappa VR, Miller WL. Rapid regulation of steroidogenesis by mitochondrial protein import. Nature 417: 87-91, 2002.

11. Melberg A, Arnell H, Dahl N, et al. Anticipation of autosomal dominant progressive external ophthalmoplegia with hypogonadism. Muscle Nerve 19: 1561-1569, 1996.

(C) 2014 The Japanese Society of Internal Medicine http://www.naika.or.jp/imonline/index.html 